\title{
Does persistent meditation and physical activity mediate the relationship between perceptions of COVID-19 and mental health? A cross sectional survey of mobile app users in the United States.
}

Jeni Green ( $\boldsymbol{\sigma}$ jeni.green@asu.du )

Arizona State University https://orcid.org/0000-0002-0218-2245

Jennifer Huberty

Arizona State University

Megan Puzia

Arizona State University

Chad Stecher

\section{Research Article}

Keywords: Coronavirus, mental health, mindfulness meditation, physical activity

Posted Date: August 28th, 2020

DOI: https://doi.org/10.21203/rs.3.rs-53798/v1

License: (c) (i) This work is licensed under a Creative Commons Attribution 4.0 International License.

Read Full License 


\section{Abstract}

Background: The Coronavirus Disease 2019 (COVID-19) pandemic has been declared an international public health emergency with potential long-lasting effects on mental health. The purpose of this survey was to examine the regional differences in mental health and perceptions of COVID-19 in light of the state-level prevalence of COVID-19 cases, estimate the associations between perceptions of COVID-19 and health behavior engagement (i.e., physical activity, mindfulness meditation) and mental health, and explore the mediating effect of health behavior engagement on the relationship between perceptions of COVID-19 and mental health.

Methods: A cross-sectional survey was distributed to a sample of US adult paying subscribers to the Calm app (data collection April 22 to June 3, 2020). The survey assessed perceptions of COVID-19, health behavior engagement, and mental health (i.e., perceived stress, post-traumatic stress disorder, and anxiety and depression). All statistical analyses were performed using R software. Differences in perceptions of COVID-19 and mental health by location were assessed using t-tests and chi-square tests. Logistic and OLS models regressed mental health and health behavior on COVID-19 perceptions, and Causal Mediation Analysis was used to estimate the significance of mediation effects.

Results: Median age ( $\mathrm{N}=8392)$ was $47(\mathrm{SD}=13.8)$ years. Mid-Atlantic region participants reported higher levels of stress, more severe depression symptoms, were more worried about COVID-19, paid more attention to COVID-19 news, and had more stress related to social distancing recommendations than participants living in other regions. The relationship between worry about COVID-19 and perceived stress was significantly mediated by changes in physical activity $(p<.001)$ and strength of meditation habit $(p<.001)$. The relationship between worry about COVID-19 and PTSD symptoms was significantly mediated by changes in physical activity $(p<.001)$ and strength of meditation habit $(p<.001)$.

Conclusions: Our findings describe the mental health impact of COVID-19 and outline how continued participation in health behaviors such as physical activity and mindfulness meditation may buffer against worsening mental health due to the COVID-19 pandemic. These data have important implications for public health agencies and health organizations to promote the maintenance of health habits to reduce the residual mental health burden of the COVID-19 pandemic.

\section{Introduction}

In January 2020, the World Health Organization declared the novel Coronavirus Disease 2019 (COVID-19) an international public health emergency,[1] and the negative mental health effects of the ongoing COVID19 pandemic are expected to be a significant, long-lasting global health problem.[2,3] In an April 2020 review of the existing literature on COVID-19 and mental health, moderate to severe levels of depressive symptoms and anxiety were reported in $16-28 \%$ of the general population and medical staff in response to the COVID-19 pandemic.[4] Additionally, fear and worry about COVID-19 is common[5] with many citing worries related to personal infection or the infection of family members, $[6,7]$ an overrun health care 
system, financial losses without recovery anytime soon,[8,9] and long-lasting isolation and movement restrictions.[10] There is previous data to suggest regional differences across the US in COVID-19 related fear and mental health (i.e., anxiety and depressive symptoms) with greater symptoms in regions with higher confirmed cases namely the Northeast New England, Northeast Mid-Atlantic, South-South Atlantic, and West Pacific regions (survey data collected March 23, 2020).[5] Based on the known mental health effects of the COVID-19 pandemic, there is a clear need for strategies to help individuals better cope with COVID-19 and mitigate the potentially long-lasting mental health consequences. Additionally, there is a need to better understand how resources for mental health should be allocated as the prevalence of COVID-19 infections changes regionally overtime.

While mental health professionals are often among the first line of treatment for poor mental health, digital approaches including mobile health (i.e., mHealth) technologies may provide a way to more widely disseminate treatment information and enable individuals to self-manage their mental health from the safety of their own home. In a recent survey $(\mathrm{N}=2,198)$ by the Academy of Medical Services, many respondents were concerned about how they would access mental health support, as many previously available in-person services have been discontinued as a result of COVID-19.[11] Stakeholders were also concerned with the capacity to handle the increased demand for mental health services and the lack of emphasis on mental health as compared to treatment of COVID-19 and its physical health impacts.[11] Importantly, self-management strategies including digital approaches to improve mental health have become an area of interest for policymakers, as many individuals fail (or are unable) to participate due to the pandemic (e.g., facility closures, reduced client load, social distancing).[12] Self-management strategies may also empower individuals by taking a more active role in their healthcare (i.e., recognizing and managing their own health problems).[13] Additionally, self-management strategies are cost-effective and can be utilized as a preventative tool (rather than prescriptive or treatment focused) that may mitigate more debilitating mental health issues from developing.[12] Common and evidence-based selfmanagement strategies for improving mental health are physical activity and mindfulness meditation, $[14,15,16]$ both of which can still be maintained during social distancing policies and stay-at-home orders, and can be adapted and delivered digitally. However, it is unknown the extent to which individuals have maintained their participation in these self-management strategies during the COVID-19 pandemic.

Physical activity has been widely adopted as a beneficial way to self-manage mental health and may attenuate the mental health decline resulting from COVID-19.[17,18] Physical activity has been shown to be as effective as antidepressants in decreasing stress, improving mood, and enhancing self-esteem. $[17,18]$ Despite the known benefits of physical activity, currently more than $60 \%$ of US adults do not engage in the recommended amount of physical activity (i.e., 150 minutes of moderate to vigorous physical activity per week) and $25 \%$ are not active at all (i.e., sedentary).[19] Social distancing, quarantine/social isolation, and closing public spaces due to COVID-19 are likely to increase these rates of physical inactivity,[20,21] and the degree to which adults change the type or duration of their physical activity during COVID-19 is also unknown. Thus, there is a need to determine how self-management strategies such as physical activity have changed as a result of the pandemic, and specifically if reductions in physical activity are associated with worsening mental health status. 
Mindfulness meditation, another self-management strategy, has also been evidenced to improve mental health and maintenance (or habituation) of this behavior, particularly during COVID-19, may be protective of worsening mental health overtime. Evidence suggests that mindfulness meditation reduces stress, improves mood (e.g., symptoms of anxiety and depression), and enhances well-being.[14,22] Though an optimal amount of mindfulness meditation has not been established, evidence suggests more benefits accrue with greater frequency of practice.[23] The habituation of mindfulness meditation practice (i.e., behavioral automaticity)[24,25] has yet to been examined in the literature[26] but may have important implications for whether individuals maintain their practice during the COVID-19 pandemic. Those with stronger meditation habits may be more likely to continue meditation practice even when daily life is disrupted, as habits are known to persist when motivation declines and other distractions are present.[25] Mindfulness meditation delivered via mHealth may be another useful way to help people maintain their practice, especially when many public facilities are closed or limit participation,[21,22,23] and popular meditation apps such as Calm or Headspace have shown promise to reduce stress and improve health. $[21,24,25,26,27,28]$ Interestingly, Calm's sales in April 2020 were $62 \%$ higher than February 2020 and $32 \%$ higher than March 2020 (unpublished sales data provided by internal Calm team), indicating that more people are accessing this type of self-management strategy in response to COVID-19. Though mHealth meditation apps have promise to help people self-manage mental health, there is a lack of understanding about how continued participation in meditation is impacted during COVID-19 and if meditation habits are associated with improved mental health.

Given the benefits of physical activity and mindfulness meditation on mental health, the purpose of this cross-sectional survey (data collected between April $22^{\text {nd }}$ to June $3^{\text {rd }} 2020$ ) was to 1 ) examine the differences in perceptions of COVID-19 (i.e., worry, attention to news, stress from social distancing) and mental health (i.e., stress, PTSD, depression, and anxiety) by region of the US, 2) explore the associations between perceptions of COVID-19 and health behavior engagement (i.e., strength of meditation habit, and changes in mindfulness meditation and physical activity) and mental health, and 3) estimate the mediating effect of health behavior engagement on the relationship between perceptions of COVID-19 and mental health. We hypothesized that 1) there would be differences in perceptions of COVID-19 and mental health in higher prevalence COVID-19 states, 2) greater negative perceptions of COVID-19 would be associated with lower health behavior engagement and greater levels of mental health, 3) health behavior engagement would mediate the relationship between of perceptions of COVID-19 and mental health.

\section{Methods}

Study Design

This is a descriptive and national longitudinal study, using a non-random convenience sample of adult paying subscribers to the mindfulness meditation mobile app, Calm. Participants of this study initially completed a cross-sectional, baseline survey called the "COVID-19 Health and Well-being Survey" and 
agreed to complete four follow-up surveys over the subsequent 12 months. The data presented in this paper come from the baseline survey administered from April $22^{\text {nd }}$ to June $3^{\text {rd }}, 2020$.

Participant Recruitment and Selection

Emails inviting Calm subscribers to participate in the study were sent on April 22 ${ }^{\text {nd }}$, April $29^{\text {th }}$, and May $6^{\text {th }}, 2020$. Subscribers were eligible if they had opened an email from Calm at least once in the last 90 days and used Calm at least once in the last 90 days, were 18 years or older, able to read and understand English, and resided in the US.

\section{Procedures}

Interested individuals were directed to a Qualtrics eligibility screener ( $\sim 1$ minute to complete). Once eligibility was determined, participants completed an electronic informed consent form and the baseline survey. There were no incentives for participation in the first wave (baseline) of the study, but incentives were offered for continued participation in subsequent waves of this study.

\section{COVID-19 Health and Well-being Survey}

The baseline survey was also administered using Qualtrics and included both investigator-developed and validated questionnaires. The investigator-developed portion of the survey included a total of 15 questions related to perceptions of COVID-19 (i.e., worry, attention to news, stress from social distancing) and 20 questions related to health behavior engagement (i.e., strength of meditation habit, and changes in mindfulness meditation and physical activity; see Table 1 for questions used in current analyses). The perceptions of COVID-19 questions were adapted from a CDC questionnaire for other infectious diseases (e.g., SARS Psychosocial Research Consortium survey).[35,36] The validated survey components assessed meditation habit strength, perceived stress, post-traumatic stress disorder (PTSD), and anxiety and depression. Demographics were collected at the end of the survey. Specifically, zip codes were used to determine participants' state of residence,[37] and states were categorized into regions according to the US Census Bureau classification system.[38] States were also designed as having a high or low prevalence of COVID-19 based on data compiled by the Center for Systems Science and Engineering at Johns Hopkins University Center describing state-level COVID-19 cases and deaths per 100,000 at the time of the initial survey distribution (April 22, 2020).[39] 


\section{Table 1. Investigator-developed survey questions used in analyses}

Prior to COVID did you meditate with Calm? [Y/N]

Do you currently meditate with Calm? [Y/N]

To what extent has COVID changed your meditation practice?

I meditate much more (5)

I meditate a little more (4)

I meditate about the same amount (3)

I meditate a little less (2)

I meditate much less (1)

I no longer meditate (0)

Prior to COVID, on average, how often did you engage in physical activity/exercise? [0-7 days/week] On average, how often do you currently engage in physical activity/exercise? [0-7 days/week] To what extent has COVID-19 changed your amount of physical activity/exercise?

- I exercise much more (5)

- I exercise a little more (4)

- I exercise about the same amount (3)

- I exercise a little less (2)

- I exercise much less (1)

- I no longer exercise (0)

Have recommendations for socially distancing caused stress for you?

Not at all (1)

A little (2)

Somewhat (3)

A lot (4)

How worried are you about...

... personally getting coronavirus?

... a family member getting coronavirus?

... the spread of coronavirus in your area?

Not at all worried (1)

A little worried (2) 
Somewhat worried (3)

A good bit worried (4)

Very worried (5)

How would you rate your attentiveness to information about ongoing changes and updates regarding the coronavirus?

Not at all paying attention to (1)

Somewhat paying attention to (2)

Moderately paying attention to (3)

Quite a bit paying attention to (4)

Very much paying attention to (5)

\section{Measures}

Perceptions of COVID-19 (i.e., worry, attention to news, stress from social distancing)

Participants were asked how worried they were about: personally getting COVID-19, a family member getting COVID-19, and the spread of COVID-19 in their area (See Table 1). Worry about COVID-19 was operationalized as the sum of responses to these three questions. Scores ranged from 3-15 where higher scores indicate greater levels of worry about COVID-19. Participants were also asked how they would rate their attentiveness to information about ongoing changes and news regarding COVID-19 (See Table 1). Scores at or below the median was used to identify individuals with low attention to COVID-19, while scores above the median are categorized as high attention to COVID-19. Finally, participants were asked if recommendations for socially distancing have caused them stress (See Table 1). Scores at or below the median were used to identify participants with low stress, scores above the median indicated high stress.

Health Behavior Engagement

\section{Mindfulness Meditation Practice}

Participants were asked about their meditation practice using Calm prior to COVID-19 as well as their current use of Calm. If participants indicated that they meditated using Calm prior to COVID-19, they were asked to what extent has COVID-19 changed their meditation practice (See Table 1). Participants who indicated they no longer participate in meditation were categorized as "stopped meditation," while all other participants were considered as "continuing meditation".

\section{Physical Activity Behavior}

Participants were asked to select how many days/week (scale of 0-7) of physical activity they participated in prior to COVID-19 as well as their current participation. Participants were also asked to 
what extent has COVID-19 changed the frequency and/or duration of physical activity (See Table 1). Changes in physical activity were calculated as the difference between the participants' reported number of days of physical activity currently and prior to COVID-19.

Validated Surveys

Self-report Behavioral Automaticity Index (SRBAI)

The SRHI is a reliable and valid measure that has demonstrated Cronbach's $\mathrm{a}=.89-.92$. Items are summed to produce a total score with higher values indicating stronger habits.[40]

Perceived Stress Scale (PSS)

The PSS includes 10 items that measure the degree of self-appraised stress in one's life within the past month.[29,30] Response items are a 5-point Likert scale from $0=$ "never" to $4=$ "very often." Items are summed to produce a total score from 0-40 with higher scores indicating higher levels of perceived stress. The PSS is a reliable and valid measure that has demonstrated good internal consistency (Cronbach's a=.74-.91)[43]

Impact of Events Scale-6 (IES-6)

The IES- 6 is a six-item abbreviated version of the IES-R (22-items) that assesses PTSD symptoms over the past seven days.[44] Response items are a 5-point Likert scale from $0=$ "not at all" to $4=$ "extremely." The score is calculated as a mean of the six items. Scores range from 0-5 with a binary cut off score of 1.75 indicating clinically important PTSD symptoms. The IES- 6 is a valid and reliable measure with excellent internal consistency $\mathrm{a}=0.91$.

Hospital Anxiety and Depression Scale (HADS)

The HADS is a 14-item scale measuring levels of anxiety and depression.[45] Seven items comprise the anxiety subscale (HADS-A) and seven items comprise the depression subscale (HADS-D). Response items are a 4-point Likert scale from 0 to 3. Items are summed to produce a total score from 0-21 on each subscale. Scores between 0-7 are considered normal, 8-10 is considered borderline abnormal, and 11-21 is considered abnormal. The HADS is a valid and reliable too with internal consistency reported to be up to $\mathrm{a}=0.93$ and $\mathrm{a}=0.90$ for the HADS-A and HADS-D subscales, respectively.

Statistical Analysis

All statistical analyses were performed using R software (version 4.0.0). Descriptive statistics were used to characterize the sample's demographics, health and the presence of chronic conditions, mental health, and COVID-19 perceptions. Differences in mental health and COVID-19 perceptions by location were assessed using t-tests and chi-square tests. Logistic and OLS models regressed mental health and health behavior on COVID-19 perceptions, and Causal Mediation Analysis from the 'mediation' package in R was used to estimate the significance of mediation effects. Specifically, the standard errors for mediation 
effects were calculated from 100 bootstrapped samples for each mediation regression. Demographic, health, and location variables were included as covariates in all regression analyses. A p-value of $<0.05$ was considered statistically significant.

\section{Results}

\section{Sample Characteristics}

The sample ( $\mathrm{N}=8392$ ) was primarily White, non-Hispanic, and female (see Table 2 ). The majority of participants had Bachelor- or graduate-level degree, were employed, and had an annual household income exceeding $\$ 100,000$. Approximately one third of participants reported having a least one medical condition associated with increased risk of severe illness from COVID-19, but over $80 \%$ perceived themselves to be in good overall health. 


\section{Table 2. Demographic and health characteristics of the sample}

Number of unique respondents, $\mathrm{N}$

Age, median (SD)

Gender ( $N=7303)$

Female, $\mathrm{n}(\%)$

Male, $\mathrm{n}(\%)$

Other, $\mathrm{n}(\%)$

Race $(\mathrm{N}=7178)$

White, n (\%)

Black/African American, n (\%)

Asian, $\mathrm{n}(\%)$

Native American/Alaska Native, $\mathrm{n}(\%)$

Native Hawaiian/Pacific Islander, n (\%)

Other, $\mathrm{n}(\%)$

Ethnicity $(\mathrm{N}=6774)$

non-Hispanic, n (\%)

Hispanic, n (\%)

People in the household, median (SD)

Income ( $\mathrm{N}=6949)$

$\$ 20,000$ or less, $n(\%)$

$\$ 21,000-\$ 40,000, n(\%)$

$\$ 41,000-\$ 60,000, n(\%)$

$\$ 61,000-\$ 80,000, n(\%)$

$\$ 81,000-\$ 100,000, n(\%)$

More than $\$ 100,000, n(\%)$

Employment $(\mathrm{N}=7297)$

Employed, n (\%)

Retired, n (\%)

Unemployed, $\mathrm{n}(\%)$
8392

$47.0(13.8)$

6129 (83.92)

$1147(15.71)$

$27(0.37)$

$6586(91.75)$

231 (3.22)

$216(3.01)$

$83(1.16)$

$27(0.38)$

$195(2.72)$

$6338(93.56)$

436 (6.44)

$2.0(1.4)$

212 (3.05)

402 (5.79)

705 (10.15)

942 (13.56)

1055 (15.18)

3633 (52.28)

5084 (69.67)

1012 (13.87)

477 (6.54) 


\begin{tabular}{|c|c|}
\hline Homemaker, n (\%) & $306(4.19)$ \\
\hline Unable to work, n (\%) & $252(3.45)$ \\
\hline Student, n (\%) & $166(2.27)$ \\
\hline \multicolumn{2}{|l|}{ Education (7319) } \\
\hline 11 th grade or less, $n(\%)$ & $8(0.11)$ \\
\hline High school or GED, n (\%) & $161(2.20)$ \\
\hline Some college, n (\%) & $826(11.29)$ \\
\hline Two-year/technical degree, n (\%) & $424(5.79)$ \\
\hline Bachelor's degree, n (\%) & $2670(36.48)$ \\
\hline Graduate degree, n (\%) & $3230(44.13)$ \\
\hline \multicolumn{2}{|l|}{ Region $(\mathrm{N}=7037)$} \\
\hline New England & $489(6.95)$ \\
\hline Mid-Atlantic & $946(13.44)$ \\
\hline East North Central & $953(13.54)$ \\
\hline West North Central & $459(6.52)$ \\
\hline South Atlantic & $1192(16.94)$ \\
\hline East South Central & $198(2.81)$ \\
\hline West South Central & $522(7.42)$ \\
\hline Mountain West & $713(10.13)$ \\
\hline Pacific West & $1565(22.24)$ \\
\hline \multicolumn{2}{|l|}{ State COVID-19 prevalence $(\mathrm{N}=7037)$} \\
\hline High-prevalence state & $3062(43.51)$ \\
\hline Low-prevalence state & 3975 (56.49) \\
\hline \multicolumn{2}{|l|}{ Health rating $(\mathrm{N}=7317)$} \\
\hline Poor, n (\%) & $1341(18.33)$ \\
\hline Good, n (\%) & $5976(81.67)$ \\
\hline \multicolumn{2}{|c|}{ Underlying medical conditions associated with increased } \\
\hline \multicolumn{2}{|l|}{ risk of severe illness $(\mathrm{N}=7335)$} \\
\hline At least one underlying medical condition, $\mathrm{n}(\%)$ & $2603(35.49)$ \\
\hline
\end{tabular}


Notes. States within each region are based on US Census Divisions. State-level COVID-19 prevalence is based on the number of COVID-19 cases per 100,000 at the time of the first survey distribution (April 22, 2020); high-prevalence states were California, Colorado, Illinois, Massachusetts, New Jersey, New York, Washington.

The underlying medical conditions associated with increased risk of severe illness from COVID-19 include asthma, cardiovascular disease, chronic lung disease, diabetes, chronic kidney disease, cancer in the past year, immunosuppressant, and hepatitis B.

The self-reported changes in preventative health behaviors are shown in Table 3, where most participants are found to have increased or maintained their physical activity and meditation habits during the initial period of the COVID-19 pandemic. There was a significant correlation between strength of meditation habit and changes in meditation during COVID-19, such that those with the strongest habits were the most likely to increase or maintain their meditation practices $(r=.37, p<.001)$.

\section{Table 3. Self-reported engagement in preventative health behaviors}

Physical activity prevalence $(\mathrm{N}=7325)$

Physical activity performance prior to COVID-19, n (\%)

$6715(91.67)$

Physical activity performance since COVID-19, n (\%)

$6468(88.30)$

Physical activity change since COVID-19 ( $N=6015)$

Increased, $\mathrm{n}(\%)$

1394 (23.18)

Maintained, $\mathrm{n}(\%)$

1585 (26.35)

Decreased, n (\%)

2537 (42.18)

Stopped, n (\%)

499 (8.30)

Meditation prevalence $(\mathrm{N}=7332)$

Meditated prior to COVID-19, n (\%)

5940 (81.01)

Meditate since COVID-19, n (\%)

$5435(74.13)$

Medication change since COVID-19 ( $\mathrm{N}=5914)$

Increased, n (\%)

$2101(35.53)$

Maintained, $\mathrm{n}(\%)$

1979 (33.46)

Decreased, n (\%)

$1479(25.01)$

Stopped, $\mathrm{n}(\%)$

$355(6.00)$

Strength of meditation habit (SRHI), mean (SD)

$10.72(4.05)$

Note. Strength of meditation habit measured using Self-Report Habit Index (SRHI). 
Mental health characteristics of the sample at the time of the baseline survey are presented in Table 4 . Compared to participants living in other parts of the country, participants living in the Mid-Atlantic (i.e., New Jersey, New York, or Pennsylvania) reported higher levels of stress and more severe depression symptoms. Participants living in the South Atlantic region (i.e., Delaware, Florida, Georgia, Maryland, North Carolina, South Carolina, Virginia, West Virginia, or District of Columbia) reported less severe depressive symptoms than participants in other regions. Participants living in states with a highprevalence of COVID-19 cases (i.e., California, Colorado, Illinois, Massachusetts, New Jersey, New York, Washington) reported more severe PTSD symptoms that did those living in states where COVID-19 was less prevalent. 
Table 4. Differences in mental health by region and state-level COVID-19 prevalence

\begin{tabular}{|c|c|c|c|c|}
\hline & $\begin{array}{l}\text { Stress, } \\
\text { mean (SD) }\end{array}$ & $\begin{array}{l}\text { PTSD, } \\
\text { mean (SD) }\end{array}$ & $\begin{array}{l}\text { Depression, } \\
\text { mean (SD) }\end{array}$ & $\begin{array}{l}\text { Anxiety, } \\
\text { mean (SD) }\end{array}$ \\
\hline \multicolumn{5}{|l|}{ Region } \\
\hline New England & $18.31(6.47)$ & $1.68(0.89)$ & $8.92(4.15)$ & $6.01(3.75)$ \\
\hline Mid-Atlantic & $18.66(6.46)^{\star \star}$ & $1.72(0.91)^{\dagger}$ & $9.13(4.22)^{\star}$ & $6.07(3.81)^{\dagger}$ \\
\hline East North Central & $17.75(6.33)$ & $1.66(0.90)$ & $8.81(4.20)$ & $5.77(3.62)$ \\
\hline West North Central & $17.92(6.29)$ & $1.65(0.87)$ & $8.86(4.09)$ & $5.97(3.82)$ \\
\hline South Atlantic & $17.81(6.31)$ & $1.63(0.87)^{\dagger}$ & $8.51(4.04)^{\star \star}$ & $5.73(3.57)$ \\
\hline East South Central & $18.76(6.87)$ & $1.67(0.93)$ & $9.15(4.43)$ & $5.92(3.70)$ \\
\hline West South Central & $17.73(6.43)$ & $1.62(0.92)$ & $8.86(4.24)$ & $5.67(3.63)$ \\
\hline Mountain West & $18.03(6.13)$ & $1.69(0.86)$ & $8.94(4.10)$ & $5.82(3.47)$ \\
\hline Pacific West & $18.03(6.14)$ & $1.70(0.87)$ & $8.88(4.06)$ & $5.88(3.50)$ \\
\hline \multicolumn{5}{|c|}{ State COVID-19 prevalence } \\
\hline High-prevalence state & $18.23(6.21)^{\dagger}$ & $1.71(0.86)^{\star \star}$ & $8.89(4.06)$ & $5.92(3.60)$ \\
\hline Low-prevalence state & $17.97(6.38)^{\dagger}$ & $1.65(0.89)^{\star \star}$ & $8.86(4.18)$ & $5.81(3.64)$ \\
\hline Total, median (SD) & $18.08(6.31)$ & $1.67(0.89)$ & $8.72(4.13)$ & $5.86(3.62)$ \\
\hline \multicolumn{5}{|l|}{${ }^{\dagger} p<.10,{ }^{*} p<.05,{ }^{* *} p<.01$} \\
\hline \multicolumn{5}{|c|}{$\begin{array}{l}\text { Notes. Stress was measured using total scores on Perceived Stress Scale, PTSD was measured using } \\
\text { mean scores on the Impact of Events Scale-6, depression and anxiety were measures using subscale } \\
\text { scores on the Hospital Depression and Anxiety Scale. }\end{array}$} \\
\hline \multicolumn{5}{|c|}{$\begin{array}{l}\text { States within each region are based on US Census Divisions. State-level COVID-19 prevalence is } \\
\text { based on the number of COVID- } 19 \text { cases per } 100,000 \text { at the time of the first survey distribution (April } \\
22,2020) \text {; high-prevalence states were California, Colorado, Illinois, Massachusetts, New Jersey, New } \\
\text { York, Washington. }\end{array}$} \\
\hline
\end{tabular}

Table 5 presents participants' reports regarding their worry about COVID-19 (i.e., getting it themselves, a family member getting it, the spread in their area), their attentiveness to news and updates regarding COVID-19, and their stress related to COVID-19 social distancing recommendations. Participants in the Mid-Atlantic were more worried about COVID-19, paid more attention to COVID-19 news and updates, and had more stress related to social distancing recommendations than participants living in other regions. Conversely, participants living in the South Atlantic reported less stress from social distancing recommendations than participants in other regions. Participants living in states where COVID-19 was 
prevalent had more COVID-19- related worry, paid more attention to COVID-19 news and updates, and experienced more stress from social distancing recommendations.

Table 5. Differences in COVID-19 perceptions by region and state-level COVID-19 prevalence

$\begin{array}{lll}\begin{array}{l}\text { Worry, mean } \\ (S D)\end{array} & \begin{array}{l}\text { High } \\ \text { attention, }\end{array} & \begin{array}{l}\text { High stress from social distancing, } \mathrm{n} \\ (\%)\end{array} \\ & \mathrm{n}(\%)\end{array}$

Region

\begin{tabular}{llll} 
New England & $9.69(2.85)$ & $155(31.70)$ & $233(47.65)$ \\
\hline Mid-Atlantic & $10.11(2.86)^{\star \star}$ & $353(37.35)^{\star}$ & $468(49.47)^{\star \star}$ \\
\hline East North Central & $9.58(2.94)$ & $312(32.74)$ & $404(42.44)$ \\
\hline West North Central & $9.55(2.78)$ & $153(33.33)$ & $213(46.41)$ \\
\hline South Atlantic & $9.59(2.92)$ & $395(33.14)$ & $494(41.44)^{\star}$ \\
\hline East South Central & $9.75(3.01)$ & $69(34.85)$ & $82(41.41)$ \\
\hline West South Central & $9.32(3.08)^{\star}$ & $162(31.03)$ & $209(40.04)^{\dagger}$ \\
\hline Mountain West & $9.41(2.99)^{\dagger}$ & $225(31.56)$ & $300(42.08)$ \\
\hline Pacific West & $9.52(2.90)$ & $544(34.85)$ & $706(45.11)$ \\
\hline $\begin{array}{l}\text { State CoVID-19 } \\
\text { prevalence }\end{array}$ & & & $1420(46.37)^{\star \star}$ \\
\hline High-prevalence state & $9.72(2.90)^{\star *}$ & $\begin{array}{l}1064 \\
(34.81)^{\star}\end{array}$ & $1832(42.92)^{\star \star}$ \\
\hline Low-prevalence state & $9.53(2.95)^{\star *}$ & $\begin{array}{l}1387 \\
(32.51)^{\star}\end{array}$ & $3252(44.37)$
\end{tabular}

${ }^{\dagger} \mathrm{p}<.10,{ }^{*} \mathrm{p}<.05,{ }^{* *} \mathrm{p}<.01$

Note. State-level COVID-19 prevalence is based on the number of COVID-19 cases per 100,000 at the time of the baseline survey (April 22, 2020); high-prevalence states were California, Colorado, Illinois, Massachusetts, New Jersey, New York, Washington.

The primary sources participants used to acquire information about COVID-19 were news media (e.g., newspapers, online newspapers, television news networks; $n=5552,75.32 \%$ ) and health officials (e.g., Centers for Disease Control, World Health Organization, state health officials; $n=5212,75.12 \%$ ). Approximately one third of participants reported acquiring information about COVID-19 from social media ( $n=2951,40.04 \%)$, doctors and medical professionals $(n=2721,36.91 \%)$, and friends and family members $(n=2543,34.50 \%)$. 
Associations between health behavior engagement and COVID-19 perceptions

Participants who were more worried about COVID-19, paid more attention to COVID-19 news and updates, and experienced more stress due to COVID-19 social distancing recommendations had greater decreases in physical activity and lower strength of meditation habit (see Table 6). Attention to news and updates about COVID-19 and stress due to social distancing recommendations was also associated with stopping meditation.

Men, White and non-Hispanic respondents, respondents with higher levels of education and higher household incomes were also less likely to decrease their engagement in physical activity. Strength of meditation habit was also generally stronger among respondents who were older, White, non-Hispanic, women, more educated, and had higher annual household incomes. Younger participants and men were more likely to report that since COVID-19, they had stopped meditating. Living in a state with high COVID19 prevalence was associated with decreases in physical activity and lower strength of meditation habit, but not with stopping their meditation practice. 
Table 6. Association between health behavior engagement and perceptions of COVID-19

Coefficient (SE)

(1)

(2)

(3)

Outcome: Changes in physical activity

COVID-19 worry

$-0.04^{\star \star}(0.01)$

COVID-19 attention

$-0.16^{\star \star}(0.06)$

Stress about social distancing

$-0.16^{\star \star}(0.05)$

Demographic Covariates

Age

$-0.01^{*}(0.002) \quad-0.003^{\dagger}(0.002) \quad-0.01^{*}(0.002)$

Racial minority status

$0.04(0.10)$

$0.07(0.10)$

$0.07(0.10)$

Male

$0.25^{\star \star}(0.07)$

$0.23^{* \star}(0.07)$

$0.24^{\star \star}(0.07)$

Hispanic

$-0.21^{\dagger}(0.11) \quad-0.27^{*}(0.11)$

$-0.26^{*}(0.11)$

High-school education only

$-0.71^{\star \star}(0.19) \quad-0.70^{\star \star}(0.19) \quad-0.69^{\star \star}(0.19)$

Undergraduate education

$-0.22^{\star \star}(0.06) \quad-0.22^{\star \star}(0.06) \quad-0.22^{* \star}(0.06)$

Income $<\$ 80,000$

$-0.28^{\star \star}(0.08) \quad-0.28^{\star \star}(0.08)$

$-0.27^{\star \star}(0.08)$

Income $\$ 81,000-\$ 100,000$

$-0.15^{\star}(0.06) \quad-0.16^{\star \star}(0.06)$

$-0.15^{\star}(0.06)$

Unemployed

$-0.08(0.09) \quad-0.08(0.09)$

$-0.07(0.09)$

Underlying medical condition

$-0.12^{*}(0.06)$

$-0.15^{\star \star}(0.06)$

$-0.16^{* \star}(0.06)$

Living in state with high COVID-19 prevalence

$-0.01^{*}(0.002)$

$-0.003^{\dagger}(0.002)$

$-0.01^{*}(0.002)$

Outcome: Stopped meditation

COVID-19 worry

$0.04^{\star}(0.02)$

COVID-19 attention

$0.36^{* *}(0.13)$

Stress about social distancing

$0.30^{*}(0.12)$

Outcome: Strength of meditation habit

COVID-19 worry

$-0.07^{\star \star}(0.02)$

COVID-19 attention

$-0.17(0.12)$

Stress about social distancing $-0.46^{* *}(0.12)$ 
${ }^{\dagger} \mathrm{p}<.10,{ }^{*} \mathrm{p}<.05,{ }^{* *} \mathrm{p}<.01$

Note. This table presents the coefficients from OLS regression models for the continuous outcomes, changes in physical activity and meditation habit strength, on COVID perceptions, and logistic regression models for the stopped meditation outcome on COIVD perceptions. Standard errors are in parentheses and are estimated using heteroscedasticity-robust procedures. The covariates listed in the first panel (age, racial minority status, female, Hispanic, high-school education only, undergraduate education, income $<\$ 80,000$, income $\$ 81,000-\$ 100,000$, unemployed, underlying medical condition, and living in state with high COVID-19 prevalence) were included in all models.

State-levelCOVID-19 prevalence is based on the number of COVID-19 cases per 100,000 at the time of the first survey distribution (April 22, 2020); high-prevalence states were California, Colorado, Illinois, Massachusetts, New Jersey, New York, Washington.

Mediation Models

Perceived stress and COVID-19 perceptions via engagement in health behaviors

The mediating effect of health behavior changes on the relationship between COVID-19 perceptions and stress is demonstrated by the regression analyses presented in Table 7. The first row and first column of Table 7 present the total effect of worry about COVID-19 on self-reported stress, and the subsequent columns illustrate how the association between worry about COVID-19 and stress is attenuated by the inclusion of each behavioral change measure. Based on bootstrapped estimation procedures, we found that the relationship between worry about COVID-19 and perceived stress was significantly mediated by changes in physical activity $(p<.001)$ and strength of meditation habit $(p<.001)$, but not significantly mediated by stopping meditation ( $p=.10 ; p$-values not displayed in Table 7). The second panel of Table 7 presents a similar mediation analysis for the relationship between attention to COVID-19 news and updates and perceived stress. The bootstrapped standard error calculations found that changes in physical activity $(p=.02)$ and stopping meditation $(p<.001)$ partially mediated the relationship between attention to COVID-19 news and updates and perceived stress, but strength of meditation habit was not a significant mediator $(p=.16)$. Finally, the relationship between perceived stress and stress due to COVID19 social distancing recommendations was significantly mediated by changes in physical activity $(p=.02)$, stopping meditation $(p<.001)$, and strength of meditation habit $(p<.001)$. 
Table 7. Mediating effect of health behavior change on stress

PSS Stress Score, Coefficient (SE)
(1)
(2)
(3)

COVID-19 worry - Total effect

Change in physical activity

$0.64^{\star \star}$

$(0.03)$

\section{COVID-19 worry - Indirect effect}

Stopped meditating

Strength of meditation habit

\section{COVID-19 attention - Total effect}

COVID-19 attention - Indirect effect

Change in physical activity

Stopped meditating

Strength of meditation habit

$0.74^{\star *}$

(0.16)

$0.60^{\star \star}$
$(0.03)$

$0.64^{* \star}$

$(0.028)$

$-0.25^{* *}$

$(0.04)$
(4) 
the continuous COVID perception measures, COVID-19 worry, COVID-19 attention, and Stress from social distancing as well as the indicated health behavior changes. Age, male, Hispanic, income $<\$ 80,000$, income $\$ 81,000-\$ 100,000$, unemployed, and underlying medical condition were included as covariates in all models, which also estimate heteroscedasticity-robust standard errors.

\section{PTSD symptoms and COVID-19 perceptions via engagement in health behaviors}

The mediating effect of health behavior changes on the relationship between COVID-19 perceptions and PTSD symptoms is outlined by the regression analyses presented in Table 8. The first row and first column of Table 8 present the total effect of worry about COVID-19 on self-reported PTSD symptoms, and the subsequent columns illustrate how the association between worry about COVID-19 and PTSD symptoms is attenuated by the inclusion of each behavioral change measure. Based on bootstrapped estimation procedures, we found that the relationship between worry about COVID-19 and PTSD symptoms was significantly mediated by changes in physical activity $(p<.001)$ and strength of meditation habit $(p<.001)$ but not significantly mediated by stopping meditation $(p=.06 ; p$-values not displayed in Table 8). The second panel of Table 8 presents the same mediation analysis for the relationship between attention to COVID-19 news and updates and PTSD symptoms, where bootstrapped standard error calculations found that changes in physical activity $(p=.02)$ and stopping meditation $(p<.001)$ significantly mediated the relationship between PTSD symptoms and attention to COVID-19 news and updates while the strength of meditation habit did not $(p=.12)$. Finally, the relationship between PTSD symptoms and stress caused by COVID-19 social distancing recommendations was partially mediated by changes in physical activity $(p=.02)$, stopping meditation $(p<.001)$, and strength of meditation habit $(p<.001)$. 
Table 8. Mediating effect of health behavior change on PTSD symptoms

PTSD Score, Coefficient (SE)
(1)
(2)
(3)
(4)

$\begin{array}{ll}\text { COVID-19 worry - Total effect } & 0.13^{\star \star} \\ & (0.003)\end{array}$

COVID-19 worry - Indirect effect

$0.13^{\star \star}$

$(0.003)$

$0.13^{* *}$

$(0.00)$

$0.13^{\star *}$

$-0.03^{* *}$

Change in physical activity

$(0.010)$

Stopped meditating

$0.23^{\star \star}$

$(0.05)$

Strength of meditation habit

$-0.01^{* *}$

$(0.003)$

COVID-19 attention - Total effect

$0.26^{* *}$

$(0.02)$

COVID-19 attention - Indirect effect

$0.26^{\star \star}(0.02)$

$0.24^{\star *}$

$(0.03)$

$(0.004)$

Change in physical activity

Change in physicalactivity

$-0.03^{\star *}$

$(0.010)$

Stopped meditating

$0.25^{\star *}$

$(0.05)$

Strength of meditation habit

$-0.02^{* *}$

$(0.003)$

\section{Stress from social distancing - Total}

effect

$0.58^{* *}$

$(0.02)$

\section{Stress from social distancing - Indirect effect}

Change in physical activity

Stopped meditating

Strength of meditation habit
$0.26^{* \star}(0.03)$ 
Note. This table presents the coefficients from OLS regression models of PTSD score on each of the continuous COVID perception measures, COVID- Age, male, Hispanic, income $<\$ 80,000$, income $\$ 81,000-\$ 100,000$, unemployed, and underlying medical condition were included as covariates in all models.

\section{Discussion}

Our findings describe the mental health impact of COVID-19 and outline how continued participation in health behaviors such as physical activity and mindfulness meditation may buffer against worsening mental health due to the COVID-19 pandemic. The aim of this baseline survey was to first examine the regional differences in mental health and perceptions of COVID-19 in light of the state-level prevalence of COVID-19 infections at the time of this survey. We additionally sought to estimate the associations between perceptions of COVID-19 and health behavior engagement and mental health, as well as explore the mediating effect of health behavior engagement on the relationship between perceptions of COVID-19 and mental health.

Mental health and perceptions of COVID-19 by region

Our findings indicate that participants living in the Mid-Atlantic region (i.e., New Jersey, New York, or Pennsylvania) had higher levels of stress and more severe depressive symptoms compared to the rest of the country, which is consistent with the fact that a high prevalence of confirmed COVID-19 cases existed in this region during the time period when this survey was administered. Participants in the Mid-Atlantic were also more worried about COVID-19, paid more attention to COVID-19 news and updates, and had more stress related to social distancing recommendations than survey participants living in other regions. Additionally, participants living in states with a high-prevalence of COVID-19 cases (i.e., California, Colorado, Illinois, Massachusetts, New Jersey, New York, Washington) reported more severe PTSD symptoms than those living in states where COVID-19 was less prevalent, and also had more COVID-19related worry, paid more attention to COVID-19 news and updates, and experienced more stress from social distancing recommendations. These preliminary findings highlight the regional differences in worry/fear about COVID-19 and mental health based on the prevalence of COVID-19 cases. As the prevalence of COVID-19 cases among regions may change over time, this information could be particularly useful for public health agencies or community health centers to direct or provide more mental health resources to those regions with high COVID-19 prevalence in an effort to mitigate the subsequent mental health burden. Additionally, future research and public health interventions should consider the local prevalence of COVID-19 and the extent to which individuals are attending to news and media to monitor news related to COVID-19 when targeting mental health interventions, as these factors may play an important role in the mental health impacts of the pandemic.

Associations of worry about COVID-19, physical activity, meditation, and mental health

Our findings suggest that higher levels of worry about COVID-19 were associated with lower physical activity levels and lower strength of meditation habits, but overall, the majority of participants reported 
increasing/maintaining their physical activity or meditation practice. Though our findings are not causal, higher levels of worry about COVID-19 may have contributed to the difficulty people had in sustaining their physical activity participation and making their meditation practice habitual. This is aligned with other research demonstrating that anxiety and worry can be detrimental to habitual participation in health-promoting behaviors, both in general[46] and in the context of COVID-19 One cross-sectional survey conducted in Belgium during their COVID-19 lockdown reported increases in physical activity but only in those who were previously low active and found decreases in physical activity in previously highly active adults.[49] Conversely, other cross-sectional studies conducted during COVID-19 reported significant declines in physical activity and increased sitting time.[35,37,38] No studies have reported patterns of meditation practice but one cross-sectional study assessing stress coping behaviors conducted in New York City based health care workers during COVID-19 reported that meditation (23\%) was a commonly endorsed behavior, along with physical activity/exercise (59\%).[52] However, physical activity and meditation patterns were not assessed prior to COVID-19. More research describing patterns of health behaviors during COVID-19 is needed to better understand how COVID-19 may contribute to long-lasting, negative health behavior changes.

Our data also suggest that higher levels of worry about COVID-19 was associated with poor mental health (i.e., increased stress and PTSD), and importantly, this relationship between worry about COVID-19 and poor mental health was mediated by higher physical activity levels and higher strength of meditation habits (similar relationships were observed for depression and anxiety; see supplementary tables). Therefore, maintaining health behaviors, particularly physical activity and meditation practice, during COVID-19 may buffer against the negative impact of COVID-19 related worry on stress, PTSD, depression and anxiety. To our knowledge this is the first study to examine the mediating effects of health behavior change, both physical activity and meditation, on the relationship between worry about COVID-19 and mental health during the pandemic. Existing research has shown that greater concern about COVID-19 was associated with greater anxiety and depression levels[53] and decreased physical activity and increased sedentary time during COVID-19 was associated with poor mental health (i.e., higher stress, anxiety, depression symptoms).[35,41] Our data and others presented here highlight the importance of maintaining health behaviors, particularly physical activity and meditation, during a pandemic to help reduce the impact of perceptions about COVID-19 on mental health. More research, especially longitudinal data, is needed to examine the long-term changes in health behaviors due to COVID-19, better disentangle the causal relationships between these psychological and behavioral outcomes, and to identify strategies for helping people maintain health promoting behaviors.

In summary, our findings support existing evidence of the beneficial health effects of physical activity and meditation on mental health outcomes.[16,55,56,57] Because the COVID-19 pandemic has profoundly impacted daily routines (e.g., social distancing, quarantine, businesses closures) and may negatively impact performance of health behaviors, it is important to continue promoting selfmanagement of health behaviors such as physical activity and meditation that can guard against worsening mental health during the COVID-19 pandemic, particularly in regions with a heightened sense of worry about COVID-19. Encouraging participation in physical activity and meditation should be an 
important public health objective during the current COVID-19 pandemic, especially because reduced physical and poor mental health has been shown to make people more susceptible to COVID-19 infection and disease transmission.[45,46] Public health agencies may consider providing strategies to help people maintain or adapt their current health behaviors. For example, digital or mHealth interventions for both physical activity and meditation have shown promise for their feasibility, scalability, and physical and mental health benefits. $[60,61,62,63]$ Digital and mHealth interventions are also convenient and often budget-friendly ways to encourage participation in both physical activity and meditation, and more research is needed to better understand their efficacy and applicability during the COVID-19 pandemic.

\section{Limitations}

Despite this study being one of the first to describe the relationship between perceptions of COVID-19, mental health, and self-management health behaviors, there are important limitations to be noted. First, our sample was primarily female, non-Hispanic, White, high-income, highly educated, and were paid subscribers of Calm, which limits the generalizability of these data. Second, this survey was crosssectional and therefore causal relationships cannot be determined from these analyses. The broader study plans to implement four more surveys over the next 12 months to provide a more comprehensive longitudinal assessment of the impact of COVID-19 on mental health and health behaviors.

\section{Conclusion}

Our findings underscore the importance of maintaining self-management health behaviors such as physical activity and meditation for sustaining one's mental health during the COVID-19 pandemic. These results suggest that public health agencies and health organizations should promote the maintenance of health habits with strategies such as digital and mHealth approaches that can be more easily adapted during stay-at-home orders and/or social distancing mandates. Future research is needed to identify the causal relationships between these psychological and behavioral outcomes and to evaluate strategies for helping people adapt their current meditation and physical activity practices for the restrictions on daily life imposed by COVID-19 public health policies.

\section{Declarations}

\section{Ethics approval and consent to participate}

The Institutional Review Board at Arizona State University (STUDY00011867) approved the study. All participants provided electronic consent.

\section{Consent to publication}

Not applicable.

\section{Availability of data and materials}


The data collected from this survey are available from the corresponding author upon reasonable request.

\section{Competing interests}

$\mathrm{JH}$ is currently the Director of Science at Calm. $\mathrm{JH}$ has been conducting research with Calm as a partner almost 5 years before becoming the Director of Science and the Scientific Advisory Board. Her role is to ensure the quality of Calm's science. There are no financial incentives from the growth of Calm for any author.

Dr. Jennifer Huberty discloses that she consultants for Calm on an as needs basis which includes directing the scientific advisory board. She does not receive any benefit from the sales of Calm and does not own stock in the company. All other authors declare no competing interests.

\section{Funding}

Not applicable.

\section{Authors' contributions}

All authors conceptualized the study and guided the study design as well as provided input in the development of relevant study outcomes. JG drafted the manuscript and JH managed JG, MP, and CS contributions to the acquisition, analysis, and interpretation of the data, and drafting of the manuscript. CS developed the analysis plan, managed the data interpretation, and drafted the manuscript. All authors critically reviewed and edited the manuscript, approved its submission, and fulfilled the criteria for authorship established by the International Committee of Medical Journal Editors.

\section{Acknowledgements}

Not applicable.

\section{References}

[1] W. H. Organization, "Mental health and psychosocial considerations during the COVID-19 outbreak." World Health Organization.

[2] A. Fiorillo and P. Gorwood, "The consequences of the COVID-19 pandemic on mental health and implications for clinical practice," European psychiatry: the journal of the Association of European Psychiatrists. 2020, doi: 10.1192/j.eurpsy.2020.35.

[3] Y. Bao, Y. Sun, S. Meng, J. Shi, and L. Lu, "2019-nCoV epidemic: address mental health care to empower society," The Lancet. 2020, doi: 10.1016/S0140-6736(20)30309-3. 
[4] R. P. Rajkumar, "COVID-19 and mental health: A review of the existing literature," Asian J. Psychiatr., p. $102066,2020$.

[5] K. M. Fitzpatrick, C. Harris, and G. Drawve, "Fear of COVID-19 and the Mental Health Consequences in America," Psychol. Trauma Theory, Res. Pract. Policy, 2020, doi: 10.1037/tra0000924.

[6] C. Wang et al., "Immediate Psychological Responses and Associated Factors during the Initial Stage of the 2019 Coronavirus Disease (COVID-19) Epidemic among the General Population in China," Int J Env. Res Public Heal., vol. 17, no. 5, 2020, doi: 10.3390/ijerph17051729.

[7] S. K. Brooks et al., "The psychological impact of quarantine and how to reduce it: rapid review of the evidence," The Lancet. 2020, doi: 10.1016/S0140-6736(20)30460-8.

[8] H. Salisbury, "Helen salisbury: Fear in the time of covid," The BMJ. 2020, doi: 10.1136/bmj.m1286.

[9] T. B et al., "Preliminary COVID-19 Fears Questionnaire: Systemic Sclerosis Chronic Medical Conditions Version 2020," 2020, doi: 10.31219/osf.io/m2ybt.

[10] B. D. Thombs et al., "Curating evidence on mental health during COVID-19: A living systematic review,” J Psychosom Res, vol. 133, p. 110113, 2020, doi: 10.1016/j.jpsychores.2020.110113.

[11] E. A. Holmes et al., "Multidisciplinary research priorities for the COVID-19 pandemic: a call for action for mental health science," The Lancet Psychiatry. 2020, doi: 10.1016/S2215-0366(20)30168-1.

[12] B. Inkster et al., "Digital health management during and beyond the COVID-19 pandemic: Opportunities, barriers, and recommendations (Preprint)," JMIR Ment. Heal., 2020, doi: 10.2196/19246.

[13] M. Lean et al., "Self-management interventions for people with severe mental illness: Systematic review and meta-analysis," British Journal of Psychiatry. 2019, doi: 10.1192/bjp.2019.54.

[14] T. M. Edenfield and S. A. Saeed, "An update on mindfulness meditation as a self-help treatment for anxiety and depression," Psychology Research and Behavior Management. 2012, doi: 10.2147/PRBM.S34937.

[15] S. L. Keng, M. J. Smoski, and C. J. Robins, "Effects of mindfulness on psychological health: A review of empirical studies," Clinical Psychology Review. 2011, doi: 10.1016/j.cpr.2011.04.006.

[16] S. A. Paluska and T. L. Schwenk, "Physical activity and mental health," Sport. Med., vol. 29, no. 3, pp. 167-180, 2000.

[17] J. Beaulac, A. Carlson, and R. J. Boyd, "Counseling on physical activity to promote mental health: Practical guidelines for family physicians," Can Fam Physician, vol. 57, no. 4, pp. 399-401, 2011.

[18] S. Saxena, M. Van Ommeren, K. C. Tang, and T. P. Armstrong, "Mental health benefits of physical activity," J. Ment. Heal., vol. 14, no. 5, pp. 445-451, 2005. 
[19] Center for Disease Control and Prevention, "Physical Activity and Health: A Report of the Surgeon General," 1999. .

[20] G. Hall, D. R. Laddu, S. A. Phillips, C. J. Lavie, and R. Arena, "A tale of two pandemics: How will COVID-19 and global trends in physical inactivity and sedentary behavior affect one another?," Progress in Cardiovascular Diseases. 2020, doi: 10.1016/j.pcad.2020.04.005.

[21] J. Burtscher, M. Burtscher, and G. P. Millet, "(Indoor) isolation, stress and physical inactivity: vicious circles accelerated by Covid-19?," Scand J Med Sci Sport., 2020, doi: 10.1111/sms.13706.

[22] J. Carmody and R. A. Baer, "Relationships between mindfulness practice and levels of mindfulness, medical and psychological symptoms and well-being in a mindfulness-based stress reduction program," J. Behav. Med., 2008, doi: 10.1007/s10865-007-9130-7.

[23] J. Lacaille, G. Sadikaj, M. Nishioka, K. Carrière, J. Flanders, and B. Knäuper, “Daily Mindful Responding Mediates the Effect of Meditation Practice on Stress and Mood: The Role of Practice Duration and Adherence," J. Clin. Psychol., 2018, doi: 10.1002/jclp.22489.

[24] W. Wood, "Habit in Personality and Social Psychology," Personal. Soc. Psychol. Rev., 2017, doi: $10.1177 / 1088868317720362$.

[25] W. Wood and D. Rünger, "Psychology of Habit," Annu Rev Psychol, vol. 67, pp. 289-314, 2016, doi: 10.1146/annurev-psych-122414-033417.

[26] V. B and O. S, "Reflections on Past Behavior: A Self-Report Index of Habit Strength.," J. Appl. Soc. Psychol., vol. 33, pp. 1313-1330, 2003.

[27] C. Behan, "The benefits of Meditation and Mindfulness practices during times of crisis such as Covid-19," Ir. J. Psychol. Med., 2020, doi: 10.1017/ipm.2020.38.

[28] J. Torous, K. Jän Myrick, N. Rauseo-Ricupero, and J. Firth, “Digital Mental Health and COVID-19: Using Technology Today to Accelerate the Curve on Access and Quality Tomorrow," JMIR Ment Heal., vol. 7, no. 3, p. e18848, 2020, doi: 10.2196/18848.

[29] X. Zhou et al., "The Role of Telehealth in Reducing the Mental Health Burden from COVID-19," Telemed. e-Health, vol. 26, no. 4, pp. 377-379, 2020, doi: 10.1089/tmj.2020.0068.

[30] J. Huberty, J. Green, C. Glissmann, L. Larkey, M. Puzia, and C. Lee, "Efficacy of the mindfulness meditation mobile app 'calm' to reduce stress among college students: Randomized controlled trial," $J$. Med. Internet Res., 2019, doi: 10.2196/14273.

[31] E. Yang, E. Schamber, R. M. L. Meyer, and J. I. Gold, "Happier Healers: Randomized Controlled Trial of Mobile Mindfulness for Stress Management," J Altern Complement Med, vol. 24, no. 5, pp. 505-513, 2018, doi: 10.1089/acm.2015.0301. 
[32] M. Economides, J. Martman, M. J. Bell, and B. Sanderson, "Improvements in Stress, Affect, and Irritability Following Brief Use of a Mindfulness-based Smartphone App: A Randomized Controlled Trial," Mindfulness (N. Y)., 2018, doi: 10.1007/s12671-018-0905-4.

[33] M. E. Puzia, J. Huberty, R. Eckert, L. Larkey, and R. Mesa, "Associations Between Global Mental Health and Response to an App-Based Meditation Intervention in Myeloproliferative Neoplasm Patients," Integr. Cancer Ther., 2020, doi: 10.1177/1534735420927780.

[34] J. Huberty et al., "Smartphone-based meditation for myeloproliferative neoplasm patients: Feasibility study to inform future trials," J. Med. Internet Res., 2019, doi: 10.2196/12662.

[35] Center for Disease Control and Prevention, "CDC COVID-19 Community Survey Question Bank (DRAFT)," U.S. National Library of Medicine, 2020. .

[36] A. M. Vartti et al., "SARS knowledge, perceptions, and behaviors: a comparison between Finns and the Dutch during the SARS outbreak in 2003," Int J Behav Med, vol. 16, no. 1, pp. 41-48, 2009, doi: 10.1007/s12529-008-9004-6.

[37] Data.gov, "Zip Codes - ZipCodes," 2017. .

[38] US Census Bureau, "Census Regions and Divisions of the United States," 2013.

[39] GitHub, "csse_covid_19_daily_reports_us," 2020. .

[40] B. Gardner, C. Abraham, P. Lally, and G. J. de Bruijn, "Towards parsimony in habit measurement: Testing the convergent and predictive validity of an automaticity subscale of the Self-Report Habit Index," Int. J. Behav. Nutr. Phys. Act., 2012, doi: 10.1186/1479-5868-9-102.

[41] S. Cohen, T. Kamarck, and R. Mermelstein, "A global measure of perceived stress.," J. Health Soc. Behav., 1983, doi: 10.2307/2136404.

[42] S. Cohen, "Perceived stress in a probability sample of the United States.," 1988.

[43] E. H. Lee, "Review of the psychometric evidence of the perceived stress scale," Asian Nurs Res (Korean Soc Nurs Sci), vol. 6, no. 4, pp. 121-127, 2012, doi: 10.1016/j.anr.2012.08.004.

[44] M. M. Hosey et al., "Screening for posttraumatic stress disorder in ARDS survivors: validation of the Impact of Event Scale-6 (IES-6)," Crit Care, vol. 23, no. 1, p. 276, 2019, doi: 10.1186/s13054-019-2553-z.

[45] A. S. Zigmond and R. P. Snaith, "The hospital anxiety and depression scale," Acta Psychiatr Scand, vol. 67, no. 6, pp. 361-370, 1983, doi: 10.1111/j.1600-0447.1983.tb09716.x.

[46] M. Banerjee, K. Cavanagh, and C. Strauss, "Barriers to Mindfulness: a Path Analytic Model Exploring the Role of Rumination and Worry in Predicting Psychological and Physical Engagement in an 
Online Mindfulness-Based Intervention," Mindfulness (N Y), vol. 9, no. 3, pp. 980-992, 2018, doi: 10.1007/s12671-017-0837-4.

[47] R. Stanton et al., "Depression, Anxiety and Stress during COVID-19: Associations with Changes in Physical Activity, Sleep, Tobacco and Alcohol Use in Australian Adults," Int J Env. Res Public Heal., vol. 17, no. 11,2020 , doi: 10.3390/ijerph17114065.

[48] F. Schuch et al., "Associations of moderate to vigorous physical activity and sedentary behavior with depressive and anxiety symptoms in self-isolating people during the COVID-19 pandemic: A crosssectional survey in Brazil," 2020.

[49] B. Constandt, E. Thibaut, V. De Bosscher, J. Scheerder, M. Ricour, and A. Willem, “Exercising in Times of Lockdown: An Analysis of the Impact of COVID-19 on Levels and Patterns of Exercise among Adults in Belgium," Int J Env. Res Public Heal., vol. 17, no. 11, 2020, doi: 10.3390/ijerph17114144.

[50] A. Ammar et al., "Effects of COVID-19 Home Confinement on Eating Behaviour and Physical Activity: Results of the ECLB-COVID19 International Online Survey," Nutrients, vol. 12, no. 6, 2020, doi: $10.3390 /$ nu12061583.

[51] J. P. Almandoz et al., "Impact of COVID-19 Stay-at-Home Orders on Weight-Related Behaviors Among Patients with Obesity," Clin. Obes., p. e12386, 2020.

[52] A. Shechter et al., "Psychological distress, coping behaviors, and preferences for support among New York healthcare workers during the COVID-19 pandemic," Gen. Hosp. Psychiatry, 2020, doi: 10.1016/j.genhosppsych.2020.06.007.

[53] B. W. Nelson, A. Pettitt, J. E. Flannery, and N. B. Allen, "Rapid Assessment of Psychological and Epidemiological Predictors of COVID-19," Int. J. Methods Psychiatr. Res., vol. 21, no. 3, pp. 169-184.

[54] B. Cheval et al., "Relationships Between Changes in Self-reported Physical Activity and Sedentary Behaviours and Health During the Coronavirus (COVID-19) Pandemic in France and Switzerland," 2020.

[55] F. J. Penedo and J. R. Dahn, "Exercise and well-being: A review of mental and physical health benefits associated with physical activity," Current Opinion in Psychiatry. 2005, doi: 10.1097/00001504200503000-00013.

[56] A. L. Rebar, R. Stanton, D. Geard, C. Short, M. J. Duncan, and C. Vandelanotte, "A meta-metaanalysis of the effect of physical activity on depression and anxiety in non-clinical adult populations," Health Psychol. Rev., 2015, doi: 10.1080/17437199.2015.1022901.

[57] D. E. Warburton, C. W. Nicol, and S. S. Bredin, "Health benefits of physical activity: the evidence," CMAJ, vol. 174, no. 6, pp. 801-809, 2006, doi: 10.1503/cmaj.051351. 
[58] D. C. Nieman and L. M. Wentz, "The compelling link between physical activity and the body's defense system," Journal of Sport and Health Science. 2019, doi: 10.1016/j.jshs.2018.09.009.

[59] J. N. Morey, I. A. Boggero, A. B. Scott, and S. C. Segerstrom, "Current directions in stress and human immune function," Current Opinion in Psychology. 2015, doi: 10.1016/j.copsyc.2015.03.007.

[60] M. Mani, D. J. Kavanagh, L. Hides, and S. R. Stoyanov, "Review and Evaluation of MindfulnessBased iPhone Apps," JMIR mHealth uHealth, 2015, doi: 10.2196/mhealth.4328.

[61] I. Plaza, M. M. P. Demarzo, P. Herrera-Mercadal, and J. García-Campayo, "Mindfulness-based mobile applications: literature review and analysis of current features," JMIR mHealth uHealth, vol. 1, no. 2, p. e24, 2013.

[62] J. Fanning, S. P. Mullen, and E. Mcauley, "Increasing physical activity with mobile devices: A metaanalysis," J. Med. Internet Res., 2012, doi: 10.2196/jmir.2171.

[63] A. M. Müller, S. Alley, S. Schoeppe, and C. Vandelanotte, "The effectiveness of e-\& mHealth interventions to promote physical activity and healthy diets in developing countries: A systematic review," International Journal of Behavioral Nutrition and Physical Activity. 2016, doi: 10.1186/s12966-016-04342.

\section{Supplementary Files}

This is a list of supplementary files associated with this preprint. Click to download.

- Covidbaselinesupplementaltables72020MEP.docx 